\title{
Natural Continuous Extensions of Runge-Kutta Methods for Volterra Integral Equations of the Second Kind and Their Applications
}

\author{
By A. Bellen, Z. Jackiewicz, R. Vermiglio, and M. Zennaro*
}

\begin{abstract}
We consider a very general class of Runge-Kutta methods for the numerical solution of Volterra integral equations of the second kind, which includes as special cases all the more important methods which have been considered in the literature. The main purpose of this paper is to define and prove the existence of the Natural Continuous Extensions (NCE's) of Runge-Kutta methods, i.e., piecewise polynomial functions which extend the approximation at the grid points to the whole interval of integration. The particular properties required of the NCE's allow us to construct the tail approximations, which are quite efficient in terms of kernel evaluations.
\end{abstract}

1. Introduction. Consider the Volterra integral equation (VIE) of the second kind

$$
y(x)=g(x)+\int_{x_{0}}^{x} k(x, s, y(s)) d s, \quad x \in\left[x_{0}, X\right],
$$

where $g:\left[x_{0}, X\right] \rightarrow R^{n}$ and $k: \Delta \times R^{n} \rightarrow R^{n}, \Delta:=\left\{(x, s): x_{0} \leq s \leq x \leq X\right\}$, are sufficiently smooth. We denote by $Y$ the unique solution of this problem.

In recent years many papers have appeared on the numerical solution of VIE's (1.1) by a Runge-Kutta (RK) type approach. Brunner, Hairer and Nørsett [8] investigated the general structure of order conditions for Volterra-Runge-Kutta (VRK) methods using the theory of $P$-series developed by Hairer [12]. The convergence and order of some classes of Runge-Kutta methods are also examined in Hairer, Lubich and Nørsett [14], and van der Houwen, Wolkenfelt and Baker [18]. Asymptotic stability analyses are given in Baker and Keech [1], Hairer and Lubich [13], and once again in [18]. Many other useful references can be found in the papers mentioned above. The recent book by Brunner and van der Houwen [10] presents the state of the art in the numerical solution of the Volterra integral and integro-differential equations, including equations with weakly singular kernels.

For VIE's the possible choice of parameters in a Runge-Kutta method is larger than for ordinary differential equations (ODE's)

$$
\left\{\begin{array}{l}
y^{\prime}(x)=f(x, y(x)), \quad x \in\left[x_{0}, X\right] \\
y\left(x_{0}\right)=y_{0}
\end{array}\right.
$$

Received March 13, 1987; revised March 11, 1988.

1980 Mathematics Subject Classification (1985 Revision). Primary 65R20; Secondary 45L10.

* The work of the first and fourth author was supported by the Italian Government from M.P.I. Funds (40\%). The work of the second author was supported by the Consiglio Nazionale delle Ricerche and by the National Science Foundation under grant NSF DMS-8520900. 
This is not surprising if one notes that (1.2) in its integrated form is equivalent to a VIE, but the contrary is, in general, not true.

There is a natural way to get RK formulas from a collocation approach (see, for example, [7], [9], [10], and Section 5 of this paper). However, many other classes of RK methods which cannot be obtained in this way and which do not seem to derive from an equally natural approach were considered in the literature (see [8], $[10],[14],[17],[18])$. They are rather formulated in a way so as to include as special cases the famous methods of Pouzet [16] and Bel'tyukov [6]. In this paper we propose a unifying notation for RK formulas for VIE's by introducing a large set of parameters. This will allow for a unique treatment of all these methods and for a development of the theory of Natural Continuous Extensions (NCE's) (see Section 3 ). Moreover, the greater number of degrees of freedom leads to the construction of methods which have better stability properties (see [4]).

Let a partition $x_{0}<x_{1}<\cdots<x_{N}=X$ of the interval of integration $\left[x_{0}, X\right]$ be given, and put $h_{n}=x_{n}-x_{n-1}, n=1,2, \ldots, N$. We consider the following very general class of $\nu$-stage VRK methods for VIE's:

$$
\left\{\begin{aligned}
Y_{i}^{(n)}= & F_{n}\left(x_{n-1}+\theta_{i} h_{n}\right) \\
& +h_{n} \sum_{j=1}^{\mu} \alpha_{i j} k\left(x_{n-1}+d_{i j} h_{n}, x_{n-1}+e_{i j} h_{n}, \sum_{l=1}^{\nu} \beta_{i j l} Y_{l}^{(n)}\right), \\
y_{n}= & \sum_{i=1}^{\nu} w_{i} Y_{i}^{(n)},
\end{aligned}\right.
$$

$i=1,2, \ldots, \nu, n=1,2, \ldots, N$, where $F_{n}(x)$ is an approximation to the tail

$$
g(x)+\int_{x_{0}}^{x_{n-1}} k(x, s, y(s)) d s
$$

This tail approximation should be chosen in such a way that it preserves the order of convergence and stability properties of the RK method and that it is as efficient as possible in terms of the number of evaluations of the kernel function $k$ (see Section 4).

Since, in general, $k(x, s, y)$ is defined only for $s \leq x$, we will always assume the kernel condition $e_{i j} \leq d_{i j}$ in (1.3).

In [1] RK formulas are examined with $\mu=\nu=p, d_{i j}=\theta_{i}, e_{i j}=\theta_{j}, \theta_{p}=1$, $\beta_{i j l}=\delta_{j l}, w_{i}=\delta_{p i}$. For the methods considered in [8], [10], and [14], which contain as special cases the methods of Pouzet and Bel'tyukov, $\mu=\nu-1=m, \alpha_{i j}=a_{i j}$, $\alpha_{m+1, j}=b_{j}, d_{m+1, j}=e_{j}, e_{i j}=c_{j}, \beta_{i j l}=\delta_{j l}, w_{i}=\delta_{m+1, i}, c_{m+1}=1$. For the methods given in [18], $\mu=\nu=m, e_{i j}=c_{j}, \beta_{i j l}=\delta_{j l}, w_{i}=\delta_{m i}, \theta_{m}=c_{m}=1$, and in [17], $\mu=\nu=m, \beta_{i j l}=\delta_{j l}, w_{i}=\delta_{m i}, \theta_{m}=e_{m m}=1, e_{m 0}=0$.

Observe that if the VRK method (1.3) is applied to the equation

$$
y(x)=y_{0}+\int_{x_{0}}^{x} f(s, y(s)) d s, \quad x \geq 0,
$$

which is equivalent to the ODE (1.2), then the resulting method is not included in the class of RK methods for (1.2). However, this method is included in the wider class of general linear methods studied by Butcher [11].

In the next section we follow the approach of Brunner, Hairer and Nørsett [8] to obtain the order conditions for the methods (1.3). In Section 3 we prove that these 
methods possess NCE's as do the RK formulas for ODE's (see [19]). This allows us, following the idea of Bellen [2], to construct quite efficient tail approximations based on composite one-step quadrature formulas. Then, in Section 5 , we briefly analyze the collocation methods for (1.1) and prove that the collocation polynomial is a NCE of the corresponding VRK method.

2. Order Conditions for VRK Methods. In this section we derive the order conditions for the methods (1.3), using a generalization of the theory of Brunner, Hairer and Nørsett [8]. We introduce the following definition.

Definition 2.1. A $\nu$-stage VRK method (1.3) for the numerical solution of the VIE (1.1) has order $p \geq 1$ (local order $p+1$ ) if on each subinterval $\left[x_{n-1}, x_{n}\right]$ the following error bound holds:

$$
\left\|y_{n}\left(x_{n}\right)-y_{n}\right\|=O\left(h_{n}^{p+1}\right), \quad h_{n} \rightarrow 0
$$

where $y_{n}(\cdot)$ is the solution of the equation

$$
z(x)=F_{n}(x)+\int_{x_{n-1}}^{x} k(x, s, z(s)) d s, \quad x \in\left[x_{n-1}, x_{n}\right] .
$$

Here, $\|\cdot\|$ stands for a suitable vector norm and the function $y_{n}$ is called a local solution on the subinterval $\left[x_{n-1}, x_{n}\right]$.

Define

$$
c_{i}=\sum_{j=1}^{\mu} \alpha_{i j}, \quad i=1,2, \ldots, \nu .
$$

Then it is easy to see that with

$$
e_{i j}=\sum_{l=1}^{\nu} \beta_{i j l} c_{l}, \quad i=1,2, \ldots, \nu, j=1,2, \ldots, \mu,
$$

the order conditions for the VRK method (1.3) applied to (1.1) are the same as the order conditions for the same method applied to the simpler equation

$$
y(x)=g(x)+\int_{x_{0}}^{x} k(x, y(s)) d s, \quad x \in\left[x_{0}, X\right] .
$$

Moreover, without loss of generality, we can restrict ourselves to the first interval $\left[x_{0}, x_{0}+h\right], h:=h_{1}$, and the method (1.3) reduces to

$$
\left\{\begin{array}{l}
Y_{i}=g\left(x_{0}+\theta_{i} h\right)+h \sum_{j=1}^{\mu} \alpha_{i j} k\left(x_{0}+d_{i j} h, \sum_{l=1}^{\nu} \beta_{i j l} Y_{l}\right) \\
y_{1}=\sum_{i=1}^{\nu} w_{i} Y_{i}
\end{array}\right.
$$

where $Y_{i}:=Y_{i}^{(1)}, i=1,2, \ldots, \nu$.

In [8] Brunner, Hairer and Nørsett have considered the equation (2.3) with $g(x) \equiv$ 0 . Since to approximate the local solution $y_{n}$ at the $n$th step $(n \geq 2)$ we have to deal with an equation of the form (2.3) in which $x_{0}$ is replaced by $x_{n-1}$ and $g$ by $F_{n}$ which, in general, is nonzero, even if $g \equiv 0$, we prefer not to adopt the simplification in [8]. Moreover, $g \not \equiv 0$ allows us to obtain the conditions on the coefficients $\theta_{i}$. 
As in [8] we transform (2.3) into an equivalent infinite system of differential equations in order to use the theory of $P$-series developed by Hairer [12]. For this purpose we set

$$
A:=\{x\} \cup\left\{a_{i}: i=0,1, \ldots\right\}, \quad a:=a_{0},
$$

and define

$$
\left\{\begin{array}{l}
y_{x}(x):=x \\
y_{a}(x):=y(x) \\
y_{a_{i}}(x):=g^{(i)}(x)+\int_{x_{0}}^{x} \frac{\partial^{i}}{\partial x^{i}} k(x, y(s)) d s,
\end{array}\right.
$$

$i=1,2, \ldots$, where $y$ is the solution of (2.3). Then

$$
\left\{\begin{array}{l}
y_{x}^{\prime}(x)=f_{x}:=1, \quad y_{x}\left(x_{0}\right)=x_{0} \\
y_{a_{i}}^{\prime}(x)=f_{a_{i}}:=\frac{\partial^{i}}{\partial x^{i}} k\left(y_{x}, y_{a}\right)+y_{a_{i+1}}, \quad y_{a_{i}}\left(x_{0}\right)=g^{(i)}\left(x_{0}\right),
\end{array}\right.
$$

$i=0,1, \ldots$ This system differs slightly from the corresponding system in [8] since we do not set $g \equiv 0$ in (2.3).

Denote by $T P$ the set of all $P$-trees and by $T P_{z}, z \in A$, the set of all $P$-trees with root index $z$ (see Brunner et al. [8] or Bellen et al. [3] for the definitions of these notions). Define also the set $T V$ of so-called Volterra trees as the smallest subset of $T P_{a}$ which satisfies the following conditions:

(i) $\varphi_{a} \in T V\left(\varphi_{a}\right.$ is the only tree of order zero in $\left.T P_{a}\right)$.

(ii) $\sigma_{k}:={ }_{a}\left[a_{1}\left[\ldots a_{k}[] \ldots\right]\right] \in T V$ for every $k \geq 0\left(\sigma_{0}={ }_{a}[]:=\tau_{a}\right.$ is the only tree of order one in $\left.T P_{a}\right)$.

(iii) If $t_{1}, \ldots, t_{m} \in T V$ then ${ }_{a}\left[\tau_{x}^{k}, t_{1}, \ldots, t_{m}\right] \in T V$ for every $k \geq 0$, where $\tau_{x}^{k}$ means that $\tau_{x}$ is repeated $k$ times.

Observe that the set $T V$ defined above is larger than the set $T V$ defined in [8]. This is due to the fact that we do not assume $g \equiv 0$ in (2.3), and hence in (2.5).

Put $y_{0}=\left(x_{0}, g\left(x_{0}\right), g^{\prime}\left(x_{0}\right), \ldots, g^{(r+1)}\left(x_{0}\right)\right)$. Let $F(t)\left(y_{0}\right)$ be an elementary differential of $f_{a}$ at $y_{0}$ corresponding to a $P$-tree $t$ with root index $a$ and an order $\rho(t)$ (see [12], [8], or [3]). We have the following theorems.

THEOREM 2.2. Consider the following equivalence relation in $T P_{a}: u \sim t$ if and only if $F(u)\left(y_{0}\right)=F(t)\left(y_{0}\right)$ for all functions $g$ and $k$ in (2.5). Then, denoting by $E(t)$ the equivalence class of $t$, we have:

(i) $E\left(\varphi_{a}\right)=\left\{\varphi_{a}\right\}$.

(ii) $E\left(\sigma_{k}\right)=\left\{\sigma_{k}\right\}, k \geq 0$.

(iii) $E\left({ }_{a}\left[\tau_{x}^{k}\right]\right)=\left\{a_{a}\left[a_{1}\left[\ldots a_{j}\left[\tau_{x}^{k-j}\right] \ldots\right]\right]: j=0,1, \ldots, k-1\right\}, k \geq 1$.

(iv) If $t={ }_{a}\left[\tau_{x}^{k}, t_{1}, \ldots, t_{m}\right]$ with $m \geq 1, t_{i} \in T V, \rho\left(t_{i}\right) \geq 1$, and $k \geq 0$, then

$$
E(t)=\bigcup_{i=0}^{k}\left\{a_{a}\left[a_{1}\left[\ldots a_{i}\left[\tau_{x}^{k-i}, u_{1}, \ldots, u_{m}\right] \ldots\right]\right]: u_{j} \in E\left(t_{j}\right)\right\} .
$$

(v) If $u, t \in T V$ and $u \neq t$ then $E(u) \neq E(t)$.

(vi) $u \notin \bigcup\{E(t): t \in T V\}$ if and only if $F(u)\left(y_{0}\right)=0$ for all functions $g$ and $k$ in (2.5).

THEOREM 2.3. For the solution $y=y_{a}$ of (2.3) we have

$$
y\left(x_{0}+h\right)=\sum_{t \in T V} \beta(t) F(t)\left(y_{0}\right) \frac{h^{\rho(t)}}{\rho(t) !},
$$


where $\beta(t)$ can be computed recursively by:

$$
\begin{aligned}
& \beta\left(\varphi_{a}\right)=1, \\
& \beta\left(\sigma_{k}\right)=1, \quad k \geq 0, \\
& \beta\left(a\left[\tau_{x}^{k}\right]\right)=k, \quad k \geq 1, \\
& \beta(t)=\frac{\rho(t)}{\rho(t)-k}(\underbrace{1, \ldots, 1}_{k \text { times }}, \rho\left(t_{1}\right), \ldots, \rho\left(t_{m}\right)) \beta\left(t_{1}\right) \cdots \beta\left(t_{m}\right) \frac{1}{k ! \mu_{1} ! \mu_{2} ! \cdots} .
\end{aligned}
$$

Here, $t={ }_{a}\left[\tau_{x}^{k}, t_{1}, \ldots, t_{m}\right], m \geq 1, \rho\left(t_{i}\right) \geq 1, k \geq 0$, and $\mu_{1}, \mu_{2}, \ldots$ are the numbers of mutually equal $P$-trees among $t_{1}, \ldots, t_{m}$.

The proofs of these theorems are similar to those given in [8] and are therefore omitted.

The expression for $y\left(x_{0}+h\right)$ appearing in Theorem 2.3 is an example of a $V$-series. To obtain order conditions for the methods (1.3), we define for any $\Phi: T V \rightarrow R$ a $V$-series as a formal power series of the form

$$
V\left(\Phi, y_{0}\right)=\sum_{t \in T V} \Phi(t) \beta(t) F(t)\left(y_{0}\right) \frac{h^{\rho(t)}}{\rho(t) !} .
$$

We are now in a position to formulate the main result of this section whose proof can be found in Bellen et al. [3].

THEOREM 2.4. Assume that

$$
\begin{gathered}
\quad \theta_{i}=c_{i}, \quad i=1,2, \ldots, \nu, \\
\sum_{l=1}^{\nu} \beta_{i j l}=1, \quad i=1,2, \ldots, \nu, j=1,2, \ldots, \mu .
\end{gathered}
$$

Then for the numerical solutions given by (2.4) we have

$$
\begin{aligned}
& Y_{i}=V\left(\Phi_{i}, y_{0}\right), \quad i=1,2, \ldots, \nu, \\
& y_{1}=V\left(\Phi, y_{0}\right),
\end{aligned}
$$

where $\Phi_{i}, \Phi: T V \rightarrow R$ are defined recursively by:

(2.10a) $\Phi_{i}\left(\varphi_{a}\right)=1$,

(2.10b) $\Phi_{i}\left(\sigma_{k}\right)=c_{i}^{k+1}, \quad k \geq 0$,

$\Phi_{i}\left({ }_{a}\left[\tau_{x}^{k}\right]\right)=\frac{1}{k}\left((k+1) \sum_{j=1}^{\mu} \alpha_{i j} d_{i j}^{k}-c_{i}^{k+1}\right), \quad k \geq 1$

$$
\Phi_{i}(t)=(\rho(t)-k) \sum_{j=1}^{\mu} \alpha_{i j} d_{i j}^{k}\left(\sum_{l=1}^{\nu} \beta_{i j l} \Phi_{l}\left(t_{1}\right)\right) \cdots\left(\sum_{l=1}^{\nu} \beta_{i j l} \Phi_{l}\left(t_{m}\right)\right)
$$

where $t={ }_{a}\left[\tau_{x}^{k}, t_{1}, \ldots, t_{m}\right], m \geq 1, \rho\left(t_{i}\right) \geq 1, k \geq 0$,

(2.10e) $\Phi(t)=\sum_{i=1}^{\nu} w_{i} \Phi_{i}(t)$

Now the order conditions for the VRK method (1.3) are expressed in the following theorem, which is a trivial consequence of (2.1), Theorem 2.3 and Theorem 2.4. 
THEOREM 2.5. The VRK method (1.3) for the numerical solution of (2.3) has order $p$ if

(i) conditions (2.7) and (2.8) hold, and

(ii) $\Phi(t)=1$ for every $t \in T V$ such that $\rho(t) \leq p$, where $\Phi$ is defined by $(2.10)$.

We have also the following corollary.

COROLlaRY 2.6. Assume that hypotheses (i)-(ii) of Theorem 2.5 are satisfied and, in addition, the condition (2.2) holds. Then the VRK method (1.3) for the numerical solution of the VIE (1.1) has order $p$.

To illustrate this theory, we have listed in Table 1 the $P$-trees from the set $T V$ and the corresponding order conditions up to order three.

TABLE 1

Order conditions for VRK methods up to order three.

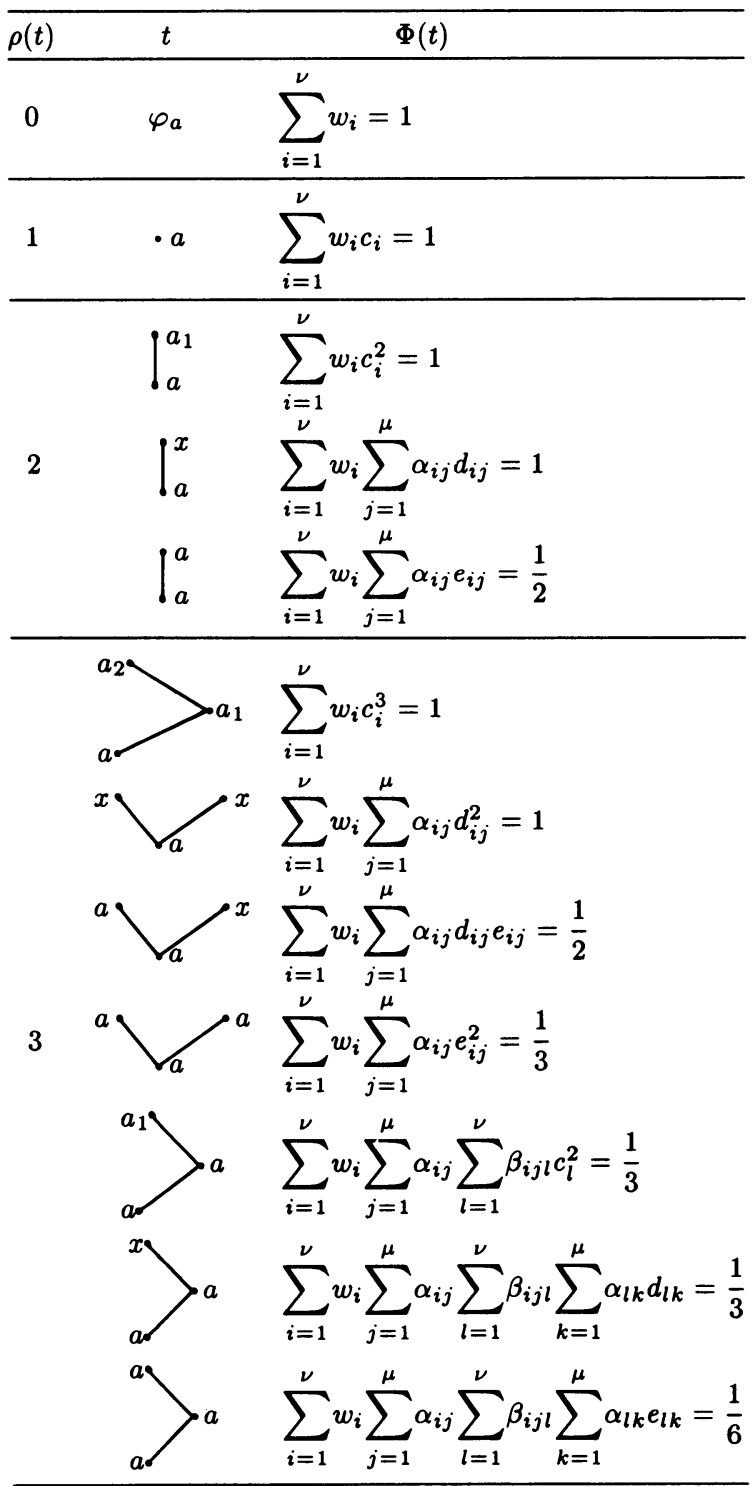


It is interesting to note that with $\mu=\nu-1=m ; \alpha_{i j}=a_{i j}, i, j=1,2, \ldots, m$; $\alpha_{m+1, j}=b_{j}, j=1,2, \ldots, m ; d_{m+1, j}=e_{j}, j=1,2, \ldots, m ; e_{i j}=c_{j}, i=$ $1,2, \ldots, m+1, j=1,2, \ldots, m ; \beta_{i j l}=\delta_{j l}, i, l=1,2, \ldots, m+1, j=1,2, \ldots, m ;$ $w_{i}=\delta_{m+1, i}, i=1,2, \ldots, m+1, c_{m+1}=1$, the VRK method (1.3) reduces to the methods considered in [8], and the corresponding order conditions for the coeffcients are the same.

3. Natural Continuous Extensions of VRK Methods. In this section we consider a $\nu$-stage VRK method (1.3) of order $p \geq 1$ and assume that all the hypotheses of Corollary 2.6 hold. For such methods, similarly as in Zennaro [19], where the RK formulas for ODE's are treated, we define and prove the existence of Natural Continuous Extensions (NCE's).

Definition 3.1. The $\nu$-stage VRK method (1.3) of order $p$ for the numerical solution of (1.1) has a NCE $u$ of degree $d$ if there exist polynomials $w_{i}, i=1,2, \ldots, \nu$, of degree less than or equal to $d$, independent of the functions $g$ and $k$, such that by putting

$$
u\left(x_{n-1}+\theta h_{n}\right):=\sum_{i=1}^{\nu} w_{i}(\theta) Y_{i}^{(n)}, \quad \theta \in[0,1]
$$

the following statements hold on each subinterval $\left[x_{n-1}, x_{n}\right]$, where $y_{n}(\cdot)$ is the local solution (see Definition 2.1):

$$
\begin{gathered}
u\left(x_{n}\right)=y_{n}, \\
\max \left\{\left\|y_{n}(x)-u(x)\right\|: x_{n-1} \leq x \leq x_{n}\right\}=O\left(h_{n}^{d+1}\right), \\
\int_{x_{n-1}}^{x_{n}} G(x)\left(y_{n}(x)-u(x)\right) d x=O\left(h_{n}^{p+1}\right)
\end{gathered}
$$

for every sufficiently smooth matrix-valued function $G$.

Remark 3.2. Observe that since, in general, $u\left(x_{n-1}\right) \neq y_{n-1}$, the NCE may have jump discontinuities at the grid points $x_{n}$.

Since $u$ is a polynomial, the condition (3.3) implies the following error bound for the derivatives of the NCE $u$ :

$$
\max \left\{\left\|y_{n}^{(k)}(x)-u^{(k)}(x)\right\|: x_{n-1} \leq x \leq x_{n}\right\}=O\left(h_{n}^{d-k+1}\right),
$$

$k=1,2, \ldots, d$, and obviously, $u^{(k)}(x)=0$ for $k \geq d+1$.

It is interesting to observe that if the NCE $u$ has degree $d=p-1$, then (3.4) follows directly from (3.3). Moreover, if $d=p$ (which, as will be shown in Theorem 3.3 , is the highest possible degree of $u$ ), then the NCE $u$ satisfies the stronger condition

$$
\int_{x_{n-1}}^{x_{n}} G(x)\left(y_{n}(x)-u(x)\right) d x=O\left(h_{n}^{p+2}\right) .
$$

We assume (2.2) and, as in the previous section, confine ourselves to studying the NCE's of the method (2.4) on the interval $\left[x_{0}, x_{0}+h\right]$. It is obvious that the following continuous version of (2.6) holds:

$$
y\left(x_{0}+\theta h\right)=\sum_{t \in T V} \beta(t) F(t)\left(y_{0}\right) \frac{(\theta h)^{\rho(t)}}{\rho(t) !} .
$$


If a NCE $u$ exists then, in view of (3.1), putting

$$
\Phi(\theta)(t):=\sum_{i=1}^{\nu} w_{i}(\theta) \Phi_{i}(t), \quad t \in T V,
$$

it is clear that for every $\theta \in[0,1]$ Theorem 2.4 holds with

$$
u\left(x_{0}+\theta h\right)=V\left(\Phi(\theta), y_{0}\right)
$$

in place of (2.9) and (3.7) in place of (2.10e). Comparing $u\left(x_{n}\right)$ and $y_{n}$, it follows that $w_{i}(1)=w_{i}, i=1,2, \ldots, \nu$; therefore, by (3.6) and (3.8), conditions (3.2), (3.3), and (3.4) are equivalent to

$$
\begin{gathered}
\Phi(1)(t)=\Phi(t), \quad t \in T V \\
\Phi(\theta)(t)=\theta^{\rho(t)}
\end{gathered}
$$

$\theta \in[0,1]$, for every $t \in T V$ such that $0 \leq \rho(t) \leq d$; and

$$
\int_{0}^{1} \theta^{r} \Phi(\theta)(t) d \theta=\frac{1}{\rho(t)+r+1}
$$

for every $t \in T V$ such that $d+1 \leq \rho(t) \leq p-1$ and $r=0,1, \ldots, p-\rho(t)-1$. Condition (3.11) should be considered only for $d \leq p-2$.

Define the integer $q:=[p / 2]$, where $[x]$ stands for the integer part of $x$, and let $\nu^{*}$ be the number of distinct values of $c_{i}, i=1,2, \ldots, \nu$. We have the following results concerning the existence of NCE's, which are similar to those given in [19].

THEOREM 3.3. If $u$ is a NCE of the $\nu$-stage VRK method (1.3) of order $p$, then its degree $d$ satisfies the condition

$$
q \leq d \leq \min \left\{\nu^{*}-1, p\right\} .
$$

Moreover, the polynomials $w_{i}, i=1,2, \ldots, \nu$, span the whole space $\Pi_{d}$ of polynomials of degree less than or equal to $d$.

Proof. Observe that (2.1), (3.2), and (3.3) imply that $d \leq p$. By (2.10a-b), (3.7), and (3.10) we have

$$
\sum_{i=1}^{\nu} w_{i}(\theta) c_{i}^{k}=\theta^{k}, \quad k=0,1, \ldots, d .
$$

Thus, after setting $C:=\left[c_{i}^{k}\right]_{i=1, k=0}^{\nu}, W^{T}:=\left[w_{i}\left(\theta_{j}\right)\right]_{i=1}^{\nu}, \underset{j=0}{d}$, and $\Theta:=\left[\theta_{j}^{k}\right]_{j, k=0}^{d}$, where $\theta_{j}, j=0,1, \ldots, d$, are distinct abscissae from the interval $[0,1]\left(0^{0}:=1\right)$, we get the system of equations

$$
W C=\Theta .
$$

From this point on, the proof proceeds as the proof of Theorem 5 in [19], using standard linear algebra arguments.

THEOREM 3.4. If the $\nu$-stage VRK method (1.3) of order p has a NCE $u$ of degree $d>q$, then it has also a NCE $\tilde{u}$ of degree $d^{\prime}$ for every $d^{\prime}=q, q+1, \ldots, d-1$.

Proof. It is easy to see that for every $n=q, q+1, \ldots, p-1$ there exists a linear projector $P_{n}: C^{0}([0,1]) \rightarrow \Pi_{n}$ such that for every continuous function $f$ the 
following conditions hold:

$$
\left\{\begin{array}{l}
P_{n} f(1)=f(1) \\
\int_{0}^{1} \theta^{r} P_{n} f(\theta) d \theta=\int_{0}^{1} \theta^{r} f(\theta) d \theta, \quad r=0,1, \ldots, p-n-2 .
\end{array}\right.
$$

The last condition should not be considered if $n=p-1$. Similarly as in the proof of Theorem 6 in [19], in view of (3.7) it follows that $\tilde{\Phi}(\theta):=\sum_{i=1}^{\nu} \tilde{w}_{i}(\theta) \Phi_{i}$, where $\tilde{w}_{i}:=P_{d} w_{i}, i=1,2, \ldots, \nu$, satisfies (3.9), (3.10), and (3.11) with $d=d^{\prime}$.

The next theorem is an analog of Theorem 7 in [19]. However, to prove it we do not adopt the strategy followed in [19] but instead we will prove the existence of a NCE $u$ of minimal degree $q$ by a suitable modification of the idea given in the proof of Theorem 10 of Bellen and Zennaro [5]. The theorem mentioned above furnishes for any RK method for ODE's a NCE which possesses some good stability properties. Hopefully, similar desirable features may be possessed also by the NCE's of VRK methods defined in this paper.

THEOREM 3.5. Every $\nu$-stage VRK method (1.3) of order p has a NCE u of minimal degree $d=q$.

Proof. Consider the matrix $A$ whose elements are

$$
a_{i l}:=\sum_{j=1}^{\mu} \alpha_{i j} \beta_{i j l}, \quad i, l=1,2, \ldots, \nu,
$$

and for all $s \geq 1$ consider the numbers $\lambda_{s n}, n=1,2, \ldots, s$, defined in Lemma 8 of [5], which satisfy the relation

$$
\sum_{n=1}^{s} \lambda_{s n} \frac{\rho !}{(\rho+n) !}=\frac{1}{\rho+s}
$$

for all $\rho \geq 0$. Then define the polynomials $w_{i}, i=1,2, \ldots, \nu$, of degree less than or equal to $q$ by

$$
\left\{\begin{array}{l}
w_{i}(1)=w_{i} \\
\int_{0}^{1} \theta^{r} w_{i}(\theta) d \theta=\sum_{n=1}^{r+1} \lambda_{r+1, n}\left[\left(A^{T}\right)^{n} w\right]_{i}, \quad r=0,1, \ldots, q-1
\end{array}\right.
$$

where $w:=\left[w_{1}, w_{2}, \ldots, w_{\nu}\right]^{T}$ and $\left[\left(A^{T}\right)^{n} w\right]_{i}$ denotes the $i$ th component of the vector $\left(A^{T}\right)^{n} w$. The last condition should not be considered for $q=0$, i.e., $p=1$, in which case the theorem is trivial. Observe that the polynomials $w_{i}, i=1,2, \ldots, \nu$, are uniquely determined by the conditions (3.15). For $q \geq 1$, i.e., $p \geq 2$ we will prove that the mappings $\Phi(\theta): T V \rightarrow R, \theta \in[0,1]$, defined by (3.7) with $w_{i}$ 's determined by (3.15a-b) satisfy (3.9), (3.10), and (3.11). Condition (3.9) is trivially satisfied as a consequence of $(3.15 \mathrm{a})$. If we prove that

$$
\int_{0}^{1} \theta^{r} \Phi(\theta)(t) d \theta=\frac{1}{\rho(t)+r+1}
$$

for $0 \leq \rho(t) \leq p-1$ and $r=0,1, \ldots, \min \{q-1, p-\rho(t)-1\}$ then, since $d=q$ (hence for $\rho(t) \geq q+1$ we have $p-\rho(t)-1 \leq q-1$ ), we will also prove (3.11). Moreover, (3.9) and (3.16) will imply (3.10). Indeed, in this case, for $\rho(t) \leq q$ the 
polynomial $\Phi(\theta)(t)-\theta^{\rho(t)}$ of degree less than or equal to $q$ lies in the kernel of $q+1$ linearly independent linear functionals on $\Pi_{q}$ and hence it must be identically zero. In order to prove (3.16), consider a $P$-tree $t \in T V$ such that $0 \leq \rho(t) \leq p-1$ and an integer $r$ such that $0 \leq r \leq \min \{q-1, p-\rho(t)-1\}$. Then (3.15b) and (3.7) yield

$$
\int_{0}^{1} \theta^{r} \Phi(\theta)(t) d \theta=\sum_{n=1}^{r+1} \lambda_{r+1, n} \sum_{i=1}^{\nu}\left[\left(A^{T}\right)^{n} w\right]_{i} \Phi_{i}(t) .
$$

For $n=1,2, \ldots, r+1$ define the $P$-tree

$$
t_{n}:=\underbrace{a[a[\ldots a}_{n \text { times }}[t] \ldots]] \in T V .
$$

We have $\rho\left(t_{n}\right)=\rho(t)+n \leq p$, and by $(2.10 \mathrm{~d}-\mathrm{e})$ it follows that

$$
\Phi\left(t_{n}\right)=\frac{(\rho(t)+n) !}{\rho(t) !} \sum_{i=1}^{\nu}\left[\left(A^{T}\right)^{n} w\right]_{i} \Phi_{i}(t) .
$$

Since by assumption the method (1.3) has order $p$, we have $\Phi\left(t_{n}\right)=1$ and hence

$$
\sum_{i=1}^{\nu}\left[\left(A^{T}\right)^{n} w\right]_{i} \Phi_{i}(t)=\frac{\rho(t) !}{(\rho(t)+n) !} .
$$

Therefore, by (3.14) and (3.17) we get (3.16), which completes the proof.

4. Tail Approximations. Different types of numerical approximations $F_{n}(x)$ to the tail have been proposed in the literature. They can be divided mainly into two classes: extended and mixed methods (compare Hairer, Lubich and Nørsett [14], van der Houwen [17], van der Houwen, Wolkenfelt and Baker [18]). In this section we define a new type of tail approximation $F_{n}(x)$ by using the NCE's introduced in the previous section. We will need the following lemma.

LEMMA 4.1. Let $u$ be a NCE (of degree d) of the VRK method (1.3) of order $p$ for the numerical solution of (1.1). Then on each subinterval $\left[x_{n-1}, x_{n}\right]$ we have

$$
\int_{x_{n-1}}^{x_{n}}\left[k\left(x, s, y_{n}(s)\right)-k(x, s, u(s))\right] d s=O\left(h_{n}^{r}\right)
$$

where

$$
r= \begin{cases}p+1 & \text { if }(3.4) \text { holds } \\ p+2 & \text { if }(3.5) \text { holds }\end{cases}
$$

Here, $y_{n}$ is the local solution defined in Section 2 (Definition 2.1).

Proof. By the smoothness of $k$ (which was assumed in Section 1) and by (3.3) we obtain

$$
\begin{aligned}
\int_{x_{n-1}}^{x_{n}} & {\left[k\left(x, s, y_{n}(s)\right)-k(x, s, u(s))\right] d s } \\
= & \int_{x_{n-1}}^{x_{n}} \frac{\partial k}{\partial y}(x, s, u(s))\left[y_{n}(s)-u(s)\right] d s+O\left(h_{n}^{2 d+3}\right) .
\end{aligned}
$$

By (3.12) we have $2 d+3 \geq 2 q+3 \geq p+2$ and the lemma follows. 
Now consider a quadrature rule of polynomial order greater than or equal to $r-2$ on the interval $[0,1]$ with weights $v_{1}, v_{2}, \ldots, v_{m}$ and abscissae $\xi_{1}, \xi_{2}, \ldots, \xi_{m}$. Then, since all the derivatives of the NCE $u$ are uniformly bounded as $h_{n} \rightarrow 0$, by (4.1) we obtain

$$
\int_{x_{n-1}}^{x_{n}} k\left(x, s, y_{n}(s)\right) d s-h_{n} \sum_{j=1}^{m} v_{j} k\left(x, x_{n-1}+\xi_{j} h_{n}, u\left(x_{n-1}+\xi_{j} h_{n}\right)\right)=O\left(h_{n}^{r}\right)
$$

where $r$ is given by (4.2).

We propose the following tail approximation:

$$
F_{n}(x)=g(x)+\sum_{k=1}^{n-1} h_{k} \sum_{j=1}^{m} v_{j} k\left(x, x_{k-1}+\xi_{j} h_{k}, u\left(x_{k-1}+\xi_{j} h_{k}\right)\right) .
$$

With this choice we have the following result concerning the global error of the method (1.3).

THEOREM 4.2. The global error of the VRK method (1.3) of order $p$ for the numerical solution of (1.1) with the tail approximation given by (4.4) satisfies

$$
\max \left\{\left\|y\left(x_{n}\right)-y_{n}\right\|: 1 \leq n \leq N\right\}=O\left(h^{r-1}\right),
$$

where $h:=\max \left\{h_{n}: 1 \leq n \leq N\right\}$ and $r$ is given by (4.2).

Proof. The proof can be carried out by standard arguments such as, for example, those given in [14], taking into account (2.1), (4.3), and (4.2).

Remark 4.3. The integer $r-1$ is the global order of the VRK method (1.3). In general it is equal to the order $p$ of the method (compare Definition 2.1). However, if the NCE $u$ satisfies the condition (3.5) then the global order is higher and is equal to the local order $p+1$.

We conclude this section by comparing the new type of tail approximation defined by (4.4) with the already known ones, for which the reader is referred to [14], [17], and [18]. In extended methods (for which $c_{\nu}=1$ ) the tail approximation $F_{n}(x)$ is built up by a composite one-step quadrature rule which on each subinterval $\left[x_{n-1}, x_{n}\right]$ is equal to that of the last stage of the method itself. This usually implies $(n-1)(\nu-1)$ kernel evaluations for each value of $x$. This is in contrast to the tail approximation (4.4), where we can choose a composite one-step Gaussian quadrature rule and hence, in general, reduce the number of kernel evaluations. Furthermore, while for our methods it is sometimes possible to attain the global order $p+1$ (see Remark 4.3), for extended methods the global order is always equal to $p$ (see Theorem 4 in [14]). In mixed methods the tail approximation $F_{n}(x)$ is constructed by means of multistep quadrature rules based only on the grid point approximations $y_{k}$. In principle, this approximation requires $n$ kernel evaluations for each value of $x$. Thus, with respect to the computational effort, the mixed methods can be more efficient than the methods (1.3), with the tail approximation given by (4.4). However, the disadvantage of this approach is that multistep quadrature rules destroy the one-step nature of the VRK methods and this can result in a loss of stability. This does not happen for the type of methods we propose (see [4]). Summing up the above discussion, we can say that the use of the tail approximation given by (4.4) results in methods which are quite efficient and possess good stability properties. 
5. Collocation Methods. In this section we treat a class of collocation methods for the numerical solution of (1.1). These methods were investigated in detail by Brunner and Nørsett [9] (see also the survey paper by Brunner [7] and the book by Brunner and van der Houwen [10] and the references contained there).

To define a collocation method, one chooses $\nu$ collocation points $0 \leq c_{1}<c_{2}<$ $\cdots<c_{\nu} \leq 1$, and defines on each subinterval $\left[x_{n-1}, x_{n}\right]$ a polynomial $\tilde{u}$ of degree $\nu-1$ by the equations

$$
\tilde{u}\left(x_{n-1}+c_{i} h_{n}\right)=g\left(x_{n-1}+c_{i} h_{n}\right)+\int_{x_{0}}^{x_{n-1}+c_{i} h_{n}} k\left(x_{n-1}+c_{i} h_{n}, s, \tilde{u}(s)\right) d s
$$

$i=1,2, \ldots, \nu$. Observe that unless $c_{1}=0$ and $c_{\nu}=1, \tilde{u}$ may have jump discontinuities at the grid points $x_{n}$.

Let quadrature rules

$$
\begin{aligned}
\int_{0}^{c_{i}} f(s) d s & \approx \sum_{j=1}^{\mu} \alpha_{i j} f\left(e_{i j}\right), \quad i=1,2, \ldots, \nu \\
\int_{0}^{1} f(s) d s & \approx \sum_{j=1}^{m} v_{j} f\left(\xi_{j}\right)
\end{aligned}
$$

be given which are of polynomial order $s_{i}, i=1,2, \ldots, \nu$, and $t$ respectively. Obviously, $s_{i} \geq \mu-1$ and $t \geq m-1$. In practice, we replace (5.1) by its discretized version

$$
\begin{aligned}
u\left(x_{n-1}+c_{i} h_{n}\right)= & F_{n}\left(x_{n-1}+c_{i} h_{n}\right) \\
& +h_{n} \sum_{j=1}^{\mu} \alpha_{i j} k\left(x_{n-1}+c_{i} h_{n}, x_{n-1}+e_{i j} h_{n}, u\left(x_{n-1}+e_{i j} h_{n}\right)\right),
\end{aligned}
$$

$i=1,2, \ldots, \nu$, where $F_{n}(x)$ is given by (4.4). Setting

$$
\begin{aligned}
& Y_{i}^{(n)}:=u\left(x_{n-1}+c_{i} h_{n}\right), \quad 1,2, \ldots, \nu, \\
& y_{n}:=u\left(x_{n-1}+h_{n}\right), \\
& \beta_{i j l}:=L_{l}\left(e_{i j}\right), \quad i, l=1,2, \ldots, \nu, j=1,2, \ldots, \mu, \\
& w_{i}:=L_{i}(1), \quad i=1,2, \ldots, \nu,
\end{aligned}
$$

where the $L_{i}$ 's are the Lagrange fundamental polynomials

$$
L_{i}(\theta):=\prod_{j=1, j \neq i}^{\nu} \frac{\left(\theta-c_{j}\right)}{\left(c_{i}-c_{j}\right)}
$$

the collocation method (5.2) takes the form (1.3) with $\theta_{i}=d_{i j}=c_{i}, i=1,2, \ldots, \nu$, and $j=1,2, \ldots, \mu$.

We will prove that the collocation polynomial $u$ is a NCE of the equivalent VRK method (1.3), provided that the polynomial orders $s_{i}$ and $t$ of the quadrature rules employed for the discretization of (5.1) are sufficiently high. We have the following theorem. 
THEOREM 5.1. Assume that the collocation points $c_{i}$ appearing in (5.2) are the abscissae of a quadrature rule of polynomial order $\delta(\delta \geq \nu-1)$. We have:

(i) If $c_{\nu}=1, s_{i} \geq \delta, i=1,2, \ldots, \nu$, and $t \geq \delta$, then the corresponding VRK method (1.3) has order $p=\delta+1, u$ is a NCE of degree $d=\nu-1$, and the global order of the method is also $\delta+1$.

(ii) If $c_{\nu}<1, s_{i} \geq \nu-2, i=1,2, \ldots, \nu$, and $t \geq \nu-1$, then the corresponding VRK method (1.3) has order $p=\nu-1, u$ is a NCE of degree $d=\nu-1$ satisfying the condition (3.5), and the global order of the method is $p+1=\nu$.

Proof. Observe first that by (5.3a) $u$ is given by (3.1) with

$$
w_{i}=L_{i}, \quad i=1,2, \ldots, \nu,
$$

where the $L_{i}$ 's are defined by $(5.5)$, and $(5.3 \mathrm{~b})$ is exactly the condition (3.2). It was shown in [9] that there exists a function $H_{n}(x, s)$ whose derivatives are uniformly bounded as $h_{n} \rightarrow 0$, such that

$$
y_{n}(x)-u(x)=r_{n}(x)+\int_{x_{n-1}}^{x} H_{n}(x, s) r_{n}(s) d s
$$

$x \in\left[x_{n-1}, x_{n}\right]$. Here, $y_{n}$ is the local solution given in Definition 2.1 and $r_{n}$ is the local defect defined by

$$
r_{n}(x):=F_{n}(x)+\int_{x_{n-1}}^{x} k(x, s, u(s)) d s-u(x) .
$$

By standard analysis we can prove that the derivatives of the collocation solution $u$ are uniformly bounded as $h_{n} \rightarrow 0$. Therefore, comparing (5.8) with (5.2), we obtain

$$
\max \left\{\left\|r_{n}\left(x_{n-1}+c_{i} h_{n}\right)\right\|: 1 \leq i \leq \nu\right\}=O\left(h_{n}^{s+2}\right)
$$

where

$$
s:=\min \left\{s_{i}: 1 \leq i \leq \nu\right\} .
$$

Thus, since $r_{n}$ is sufficiently smooth, we get

$$
\max \left\{\left\|r_{n}(x)\right\|: x_{n-1} \leq x \leq x_{n}\right\}=O\left(h_{n}^{\min \{\nu, s+2\}}\right),
$$

and, by (5.7),

$$
\max \left\{\left\|y_{n}(x)-u(x)\right\|: x_{n-1} \leq x \leq x_{n}\right\}=O\left(h_{n}^{\min \{\nu, s+2\}}\right) .
$$

Moreover, if $G$ is a sufficiently smooth matrix-valued function, then in view of (5.7) we obtain

$$
\int_{x_{n-1}}^{x_{n}} G(x)\left(y_{n}(x)-u(x)\right) d x=\int_{x_{n-1}}^{x_{n}} R_{n}(x) r_{n}(x) d x
$$

where

$$
R_{n}(x):=G(x)+\int_{x}^{x_{n}} G(s) H_{n}(s, x) d s .
$$

(i) Since $c_{\nu}=1$ and, by (5.10), $s \geq \delta,(5.3 \mathrm{~b}),(5.7)$, and (5.9) yield

$$
y_{n}\left(x_{n}\right)-y_{n}=\int_{x_{n-1}}^{x_{n}} H_{n}\left(x_{n}, s\right) r_{n}(s) d s+O\left(h_{n}^{\delta+2}\right) .
$$


On the other hand, the $c_{i}$ 's are the abscissae of a quadrature rule of polynomial order $\delta$ on $[0,1]$, and thus, by (5.9) and (5.13), we get

$$
\left\|y_{n}\left(x_{n}\right)-y_{n}\right\|=O\left(h_{n}^{\delta+2}\right)
$$

which means that $p=\delta+1$. Moreover, since $s+2 \geq \delta+2 \geq \nu+1$, (5.11) implies (3.3) with $d=\nu-1$, while as before, (5.9) and (5.12) imply (3.4). Finally, in view of (4.2), $t \geq \delta=p-1=r-2$, and it follows from Theorem 4.2 that the global order of the method is $p=\delta+1$.

(ii) Since $c_{\nu}<1$ we cannot take advantage of (5.9) to estimate the order of the method. However, since by (5.10) we have $s \geq \nu-2$, the relation (5.11) yields

$$
\left\|y_{n}\left(x_{n}\right)-y_{n}\right\|=O\left(h_{n}^{\nu}\right)
$$

which means that the order of the method (1.3) is $p=\nu-1$. We have also (3.3) with $d=\nu-1$ and (3.5). Finally, in view of (4.2), $t \geq \nu-1=p=r-2$, and it follows from Theorem 4.2 that the global order of the method (1.3) is $p+1=\nu$.

Dipartimento di Scienze Matematiche

Università di Trieste

I-34100 Trieste, Italy

Department of Mathematics

Arizona State University

Tempe, Arizona 85207

Dipartimento di Matematica e Informatica

Università di Udine

I-33100 Udine, Italy

Dipartimento di Matematica e Informatica

Università di Udine

I-33100 Udine, Italy

1. C. T. H. BAKER \& M. S. KEECH, "Stability regions in the numerical treatment of Volterra integral equations," SIAM J. Numer. Anal., v. 15, 1978, pp. 394-417.

2. A. BELLEN, "Constrained mesh methods for functional differential equations," in Delay Equations, Approximation and Application (G. Meinardus \& G. Nürnberger, eds.), Internat. Ser. Numer. Math., vol. 74, Birkhäuser Verlag, Basel, 1985, pp. 52-70.

3. A. Bellen, Z. JaCkiewicz, R. Vermiglio \& M. Zennaro, Natural Continuous Extensions of Runge-Kutta Methods for Volterra Integral Equations of the Second Kind and Their Applications, Report 65R20-7, University of Arkansas, Fayetteville, 1987.

4. A. Bellen, Z. Jackiewicz, R. Vermiglio \& M. Zennaro, Stability Analysis of RungeKutta Methods for Volterra Integral Equations of Convolution Type, Report 107, Dept. of Math., Arizona State University, Tempe, 1988.

5. A. BELLEN \& M. ZENNARO, "Stability properties of interpolants for Runge-Kutta methods," SIAM J. Numer. Anal., v. 25, 1988, pp. 411-432.

6. B. A. BEL'TYUKOV, "An analog of the Runge-Kutta method for solution of nonlinear Volterra type integral equations," Differential Equations, v. 1, 1965, pp. 417-426.

7. H. BRUNNER, "Collocation methods for one-dimensional Fredholm and Volterra integral equations," in The State of the Art in Numerical Analysis (A. Iserles \& M. J. D. Powell, eds.), Clarendon Press, Oxford, 1987, pp. 563-600.

8. H. BRUNNER, E. HAIRER \& S. P. NøRSETT, "Runge-Kutta theory for Volterra integral equations of the second kind," Math. Comp., v. 39, 1982, pp. 147-163.

9. H. BRUNNER \& S. P. NøRSETT, "Superconvergence of collocation methods for Volterra and Abel integral equations of the second kind," Numer. Math., v. 36, 1981, pp. 347-358. 
10. H. BRUNNER \& P. J. VAN DER HOUWEN, The Numerical Solution of Volterra Equations, North-Holland, Amsterdam, 1986.

11. J. C. ButCHER, The Numerical Analysis of Ordinary Differential Equations: Runge-Kutta and General Linear Methods, Wiley, Chichester, New York, 1987.

12. E. HAIRER, "Order conditions for numerical methods for partitioned ordinary differential equations," Numer. Math., v. 36, 1981, pp. 431-445.

13. E. HAIRER \& C. LUBICH, "On the stability of Volterra-Runge-Kutta methods," SIAM J. Numer. Anal., v. 21, 1984, pp. 123-135.

14. E. HAIRER, C. LUBICH \& S. P. NøRSETT, "Order of convergence of one-step methods for Volterra integral equations of the second kind," SIAM J. Numer. Anal., v. 20, 1983, pp. 569-579.

15. S. P. NøRSETT \& G. WANNER, "The real-pole sandwich for rational approximations and oscillation equations," BIT, v. 19, 1979, pp. 79-94.

16. P. POUzET, "Etude en vue de leur traitement numérique des équations intégrales de type Volterra," Rev. Française Traitement Information (Chiffres), v. 6, 1963, pp. 79-112.

17. P. J. VAN DER HOUWEN, "Convergence and stability results in Runge-Kutta type methods for Volterra integral equations of the second kind," $B I T$, v. 20, 1980, pp. 375-377.

18. P. J. VAN Der HouWen, P. H. M. Wolkenfelt \& C. T. H. BAKER, "Convergence and stability analysis for modified Runge-Kutta methods in the numerical treatment of second-kind Volterra integral equations," IMA J. Numer. Anal., v. 1, 1981, pp. 303-328.

19. M. ZENNARO, "Natural continuous extensions of Runge-Kutta methods," Math. Comp., v. 46, 1986, pp. 119-133. 